\title{
Long-Term Response of Classic Kaposi's Sarcoma to Intralesional Doxorubicin: A Case Report
}

\author{
Yasir Azim Mirza Davide Altamura Torkan Hirbod Roberto Verdolini \\ Department of Dermatology, Princess Alexandra Hospital NHS Trust, Harlow, UK
}

\section{Key Words}

Classic Kaposi's sarcoma · Kaposi's sarcoma - Intralesional doxorubicin · Treatment

\begin{abstract}
Classic Kaposi's sarcoma (KS) is a disease with low mortality but high morbidity. The optimum treatment of KS depends upon several factors, including location of lesions, disease progression, severity of symptoms and patient preference. We report the long-term response to the use of intralesional doxorubicin to successfully treat a large cutaneous lesion of KS on a patient refractory to traditional treatments.

(c) 2015 S. Karger AG, Basel
\end{abstract}

\section{Case Presentation}

An HIV-negative male, in his sixties, originally presented with a 6-month history of an isolated large nodule on the right malleolar region which was confirmed as Kaposi's sarcoma (KS) on excision. Subsequently he continued to develop more papules and plaques on the feet and over the following 7 years underwent excision of around 20 skin lesions under local anaesthesia. More recently he developed, simultaneously, a crop of 5 nodules and a large plaque of KS $5.5 \mathrm{~cm}$ in diameter on the dorsum of the right foot with extension to the interdigital spaces and toes (fig. 1a, fig. 2a). He declined radiotherapy due to his difficulty in attending the radiotherapy centre. Chemotherapy with intravenous liposome-encapsulated doxorubicin was not considered because of the significant comorbidities including mild to moderate heart failure. Therefore, in the following order, we tried two courses of photodynamic therapy, imiquimod 5\% cream applied once daily for 8 weeks and a cycle of antiherpes therapy based on valganciclovir. No response was documented. As a result it was decided to administer intralesional vinblastine $1 \mathrm{mg} / \mathrm{ml}$ diluted 1:5 with saline solution. 
Mirza et al.: Long-Term Response of Classic Kaposi's Sarcoma to Intralesional Doxorubicin: A Case Report

However, the patient developed side effects such as general malaise, swelling and erythema of the perioral and periocular areas and tingly tongue after the first injection, in keeping with a possible allergic reaction. At that point, at a bit of a loss for options, we switched to doxorubicin. Following a serial dilution test from 1:20 to undiluted, this medication was used intradermally at the dose of $2 \mathrm{mg} / \mathrm{ml}$ diluted 1:1 with saline solution. The large KS plaque on the dorsum of the right foot was treated by spacing the intradermal injections every $5 \mathrm{~mm}$, using a similar technique to that used in the treatment of axillary hyperhidrosis with botulinum A toxin. About $0.2 \mathrm{ml}$ was used for each site. The treatment led to complete resolution of the KS lesions after 4 treatments spaced 6 weeks apart. We observed skin necrosis in some well-delimited areas following each injection (fig. 1b), associated with mild to moderate pain which was well controlled with paracetamol 1,000 mg twice daily. The treatment caused no systemic side effects. The patient has not developed any new lesions for almost 18 months and continues to remain clear (fig. 1c, fig. 2b).

\section{Discussion}

$\mathrm{KS}$ is an angioproliferative disease associated with human herpesvirus 8 [1]. The classic variant of KS is characterised by a low mortality and significant morbidity, and treatment needs to be finely balanced between these two aspects [2]. To date, a curative treatment has yet to be found and therapeutic management includes surgery, radiotherapy, chemotherapy or a combination of all of the above [2].

Although in recent years numerous agents such as thalidomide, imatinib, sirolimus as well as matrix metalloproteinase inhibitors (COL-3), anti-angiogenic factors (bevacizumab) and NF- $\kappa \mathrm{B}$ inhibitors (bortezomib) have been tried, the outcome of these treatments remains unsatisfactory. Liposomal anthracyclines (pegylated or liposomal daunorubicin or doxorubicin) and taxanes such as paclitaxel therefore remain the cornerstones of systemic therapy against KS. Doxorubicin acts through intercalation of double-stranded DNA bases and by inhibiting DNA topoisomerase II whose activity is markedly increased in proliferating cells. It represents a particularly effective treatment for widespread KS as it accumulates in highly vascularised lesions.

Common agents for intralesional use in KS include interferon alpha [3], and more recently vincristine and vinblastine [4]. They lead to regression with temporary success rates of up to $88 \%$. The therapeutic effects of these agents last about 4 months [4]. Although intralesional doxorubicin is a standard treatment for KS in some centres, there are no publications showing the effectiveness of this treatment in KS.

Intralesional doxorubicin was very successful in this case and the persistent remission induced would suggest that doxorubicin might be equally or even more effective than other standard intralesional treatments for KS cutaneous lesions in selected cases. Further studies with a large cohort of patients could be helpful to clarify the impact of intralesional doxorubicin in KS.

\section{References}

1 Moore PS, Chang Y: Detection of herpesvirus-like DNA sequences in Kaposi's sarcoma in patients with and without HIV infection. N Engl J Med 1995;332:1181-1185.

-2 Régnier-Rosencher E, Guillot B, Dupin N: Treatments for classic Kaposi sarcoma: a systematic review of the literature. J Am Acad Dermatol 2013;68:313-331. 
Mirza et al.: Long-Term Response of Classic Kaposi's Sarcoma to Intralesional Doxorubicin: A Case Report

3 Brambilla L, Bellinvia M, Tourlaki A, Scoppio B, Gaiani F, Boneschi V: Intralesional vincristine as first-line therapy for nodular lesions in classic Kaposi sarcoma: a prospective study in 151 patients. Br J Dermatol 2010;162:854-859.

-4 Trattner A, Reizis Z, David M, Ingber A, Hagler J, Sandbank M: Therapeutic effect of intralesional interferon in classical Kaposi's sarcoma. Br J Dermatol 1993;129:590-593.
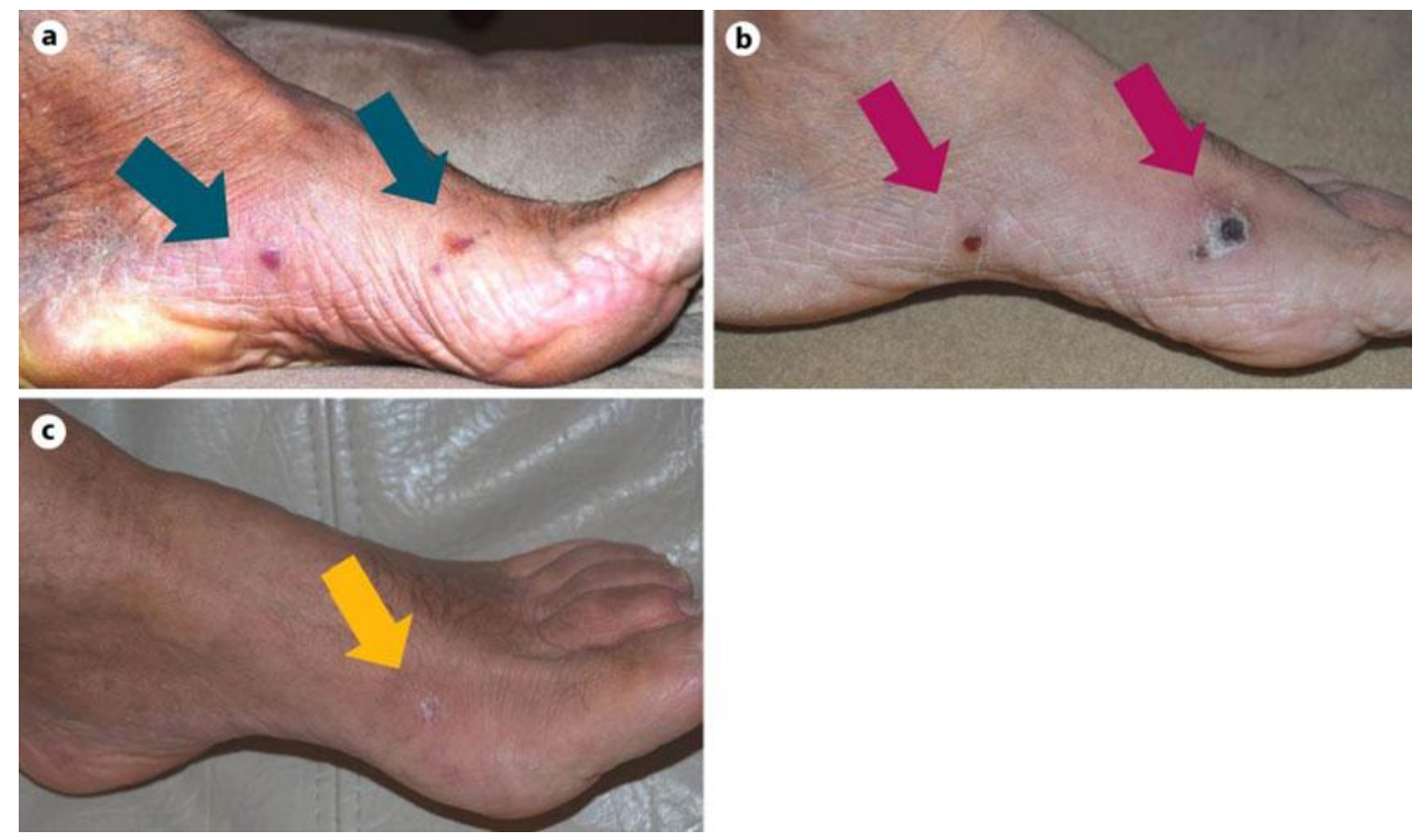

Fig. 1. a Nodules on the dorsum of the foot (blue arrows) before treatment with intralesional doxorubicin. b The same lesions (red arrows) 2 weeks after the first injection with intralesional doxorubicin. c After over a year from the beginning of treatment, only some minimal scarring (yellow arrow) was identifiable.
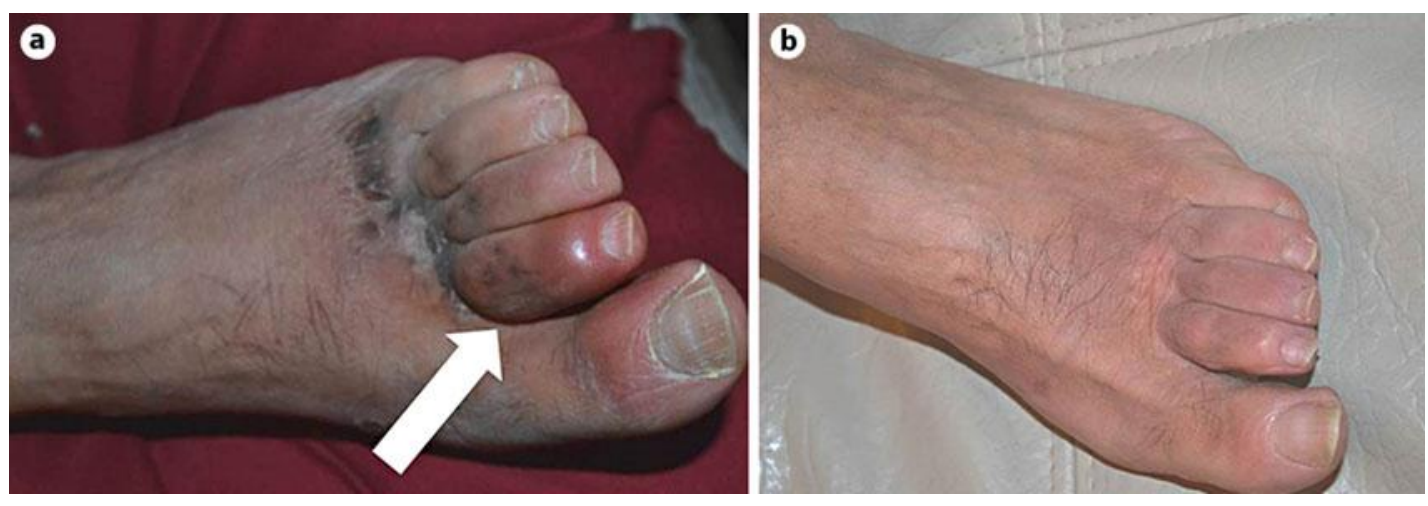

Fig. 2. a Before the treatment the second toe was swollen and painful (white arrow), with significant impact on the patient's activities. b After a year an area of mild post-inflammatory hyperpigmentation remained the only visible residual consequence of the treatment. 